\title{
THE Design of ACTIVE Controller For THE OUTPUT REGULATION OF LIU-LIU-LIU-SU ChaOtic SYSTEM
}

\author{
Sundarapandian Vaidyanathan ${ }^{1}$ \\ ${ }^{1}$ Research and Development Centre, Vel Tech Dr. RR \& Dr. SR Technical University \\ Avadi, Chennai-600 062, Tamil Nadu, INDIA \\ sundarvtuegmail.com
}

\begin{abstract}
This paper derives new results on the active controller design for the output regulation problem of the LiuLiu-Liu-Su chaotic system (2006), which is one of the paradigms of 3-dimensional chaotic systems discovered by C. Liu, T. Liu, L. Liu and Y. Su. Explicitly, for the constant tracking problem, new state feedback control laws have been derived for regulating the output of the Liu-Liu-Liu-Su chaotic system. The output regulation of the Liu-Liu-Liu-Su chaotic system has important applications in many areas of Science and Engineering. Numerical simulations are shown to validate and demonstrate the effectiveness of the control schemes derived in this paper for the output regulation of the Liu-Liu-Liu-Su system.
\end{abstract}

\section{KEYWORDS}

Active Control; Chaos; Liu-Liu-Liu-Su system; Nonlinear Control Systems; Output Regulation.

\section{INTRODUCTION}

In control systems literature, state feedback stabilization and output regulation are some of the core research problems, which have important applications in Science and Engineering. Basically, the output regulation problem is to control a fixed linear or nonlinear plant so that the output of the plant tracks reference signals produced by the exo-system. For linear control systems, the output regulation problem has been solved by Francis and Wonham ([1], 1975). For nonlinear control systems, the output regulation problem was solved by Byrnes and Isidori ([2], 1990) generalizing the internal model principle obtained in [1]. Using Centre Manifold Theory [3], Byrnes and Isidori derived regulator equations, which characterize the solution of the output regulation problem of nonlinear control systems with some stability assumptions.

The output regulation of nonlinear control systems has been studied well in the last two decades [4-14]. In [4], Mahmoud and Khalil obtained results on the asymptotic regulation of minimum phase nonlinear systems using output feedback. In [5], Fridman solved the output regulation problem for nonlinear control systems with delay using centre manifold theory. In [6-7], Chen and Huang obtained results on the robust output regulation for output feedback systems with nonlinear exosystems. In [8], Liu and Huang obtained results on the global robust output regulation problem for lower triangular nonlinear systems with unknown control direction.

DOI : $10.5121 /$ ijcsea.2012.2312 
In [9], Immonen obtained results on the practical output regulation for bounded linear infinitedimensional state space systems. In [10], Pavlov, Van de Wouw and Nijmeijer obtained results on the global nonlinear output regulation using convergence-based controller design. In [11], Xi and Dong obtained results on the global adaptive output regulation of a class of nonlinear systems with nonlinear exosystems. In [12-14], Serrani, Isidori and Marconi obtained results on the semiglobal and global output regulation problem for minimum-phase nonlinear systems.

In this paper, new results have been derived on the active controller design for the output regulation problem for the Liu-Liu-Liu-Su chaotic system ([15], 2006). Explicitly, we find state feedback control laws solving the constant regulation problem of the Liu-Liu-Liu-Su chaotic system using the regulator equations of Byrnes and Isidori (1990). The Liu-Liu-Liu-Su chaotic system is a classical three-dimensional chaotic system discovered by C. Liu, T. Liu, L. Liu and Y. Su. It has important applications in Science and Engineering.

This paper is organized as follows. Section 2 gives a brief review the problem statement of output regulation problem for nonlinear control systems and the regulator equations of Byrnes and Isidori [2]. Section 3 describes the main results of this paper, namely, the solution of the output regulation problem for the Liu-Liu-Liu-Su chaotic system for the important case of constant reference signals (set-point signals). Section 4 describes the numerical simulations for the state feedback controllers solving the output regulation problem for the Liu-Liu-Liu-Su chaotic system. Section 5 contains a summary of the main results derived in this paper.

\section{OUtPut Regulation Problem For Nonlinear Control Systems}

In this section, we consider a multi-variable nonlinear control system described by

$$
\begin{aligned}
& \dot{x}=f(x)+g(x) u+p(x) \omega \\
& \dot{\omega}=s(\omega) \\
& e=h(x)-q(\omega)
\end{aligned}
$$

Here, the differential equation (1a) describes the plant dynamics with state $x$ defined in a neighbourhood $X$ of the origin of $R^{n}$ and the input $u$ takes values in $R^{m}$ subject to te effect of a disturbance represented by the vector field $p(x) \omega$. The differential equation (1b) describes an autonomous system, known as the exosystem, defined in a neighbourhood $W$ of the origin of $R^{k}$, which models the class of disturbance and reference signals taken into consideration. The equation (2) defines the error between the actual plant output $h(x) \in R^{p}$ and a reference signal $q(\omega)$, which models the class of disturbance and reference signals taken into consideration.

We also assume that all the constituent mappings o the system (1) and the error equation (2), namely, $f, g, p, s, h$ and $q$ are continuously differentiable mappings vanishing at the origin, i.e.

$$
f(0)=0, g(0)=0, p(0)=0, s(0)=0, h(0)=0 \text { and } q(0)=0 .
$$

Thus, for $u=0$, the composite system (1) has an equilibrium $(x, \omega)=(0,0)$ with zero error (2).

A state feedback controller for the composite system (1) has the form 
International Journal of Computer Science, Engineering and Applications (IJCSEA) Vol.2, No.3, June 2012

$$
u=\rho(x, \omega)
$$

where $\rho$ is a continuously differentiable mapping defined on $X \times W$ such that $\rho(0,0)=0$.

Upon substitution of the feedback control law (3) into (1), we get the closed-loop system

$$
\begin{aligned}
& \dot{x}=f(x)+g(x) \rho(x, \omega)+p(x) \omega \\
& \dot{\omega}=s(\omega)
\end{aligned}
$$

The purpose of designing the state feedback controller (3) is to achieve both internal stability and output regulation of the given nonlinear control system (1). Formally, we can summarize these requirements as follows.

\section{State Feedback Regulator Problem [2]:}

Find, if possible, a state feedback control law $u=\rho(x, \omega)$ such that the following conditions are satisfied.

(OR1) [Internal Stability] The equilibrium $x=0$ of the dynamics

$$
\dot{x}=f(x)+g(x) \rho(x, 0)
$$

is locally exponentially stable.

(OR2) [Output Regulation] There exists a neighbourhood $U \subset X \times W$ of $(x, \omega)=(0,0)$ such that for each initial condition $(x(0), \omega(0)) \in U$, the solution $(x(t), \omega(t))$ of the closed-loop system (4) satisfies

$$
\lim _{t \rightarrow \infty}[h(x(t))-q(\omega(t))]=0 .
$$

Byrnes and Isidori [2] solved the output regulation problem stated above under the following two assumptions.

(H1) The exosystem dynamics $\dot{\omega}=s(\omega)$ is neutrally stable at $\omega=0$, i.e. the exosystem is Lyapunov stable in both forward and backward time at $\omega=0$.

(H2) The pair $(f(x), g(x))$ has a stabilizable linear approximation at $x=0$, i.e. if

$$
A=\left[\frac{\partial f}{\partial x}\right]_{x=0} \text { and } B=\left[\frac{\partial g}{\partial x}\right]_{x=0} \text {, }
$$

then $(A, B)$ is stabilizable.

Next, we recall the solution of the output regulation problem derived by Byrnes and Isidori [2]. 
Theorem 1. [2] Under the hypotheses (H1) and (H2), the state feedback regulator problem is solvable if and only if there exist continuously differentiable mappings $x=\pi(\omega)$ with $\pi(0)=0$ and $u=\varphi(\omega)$ with $\varphi(0)=0$, both defined in a neighbourhood of $W^{0} \subset W$ of $\omega=0$ such that the following equations (called the regulator equations) are satisfied:

(1) $\frac{\partial \pi}{\partial \omega} s(\omega)=f(\pi(\omega))+g(\pi(\omega)) \varphi(\omega)+p(\pi(\omega)) \omega$

(2) $h(\pi(\omega))-q(\omega)=0$

When the regulator equations (1) and (2) are satisfied, a control law solving the state feedback regulator problem is given by

$$
u=\varphi(\omega)+K[x-\pi(\omega)]
$$

where $K$ is any gain matrix such that $A+B K$ is Hurwitz.

\section{Output Regulation OF The LiU-LiU-Liu-Su Chaotic System}

In this section, we solve the output regulation problem for the Liu-Liu-Liu-Su system ([15], 2006), which is one of the paradigms of the 3-dimensional chaotic systems described by the dynamics

$$
\begin{aligned}
& \dot{x}_{1}=a\left(x_{2}-x_{1}\right) \\
& \dot{x}_{2}=b x_{1}-x_{1} x_{3}+x_{1}^{2}+u \\
& \dot{x}_{3}=-c x_{3}+x_{1}^{2}
\end{aligned}
$$

where $x_{1}, x_{2}, x_{3}$ are the states of the system, $a, b, c$ are positive, constant parameters of the system and $u$ is the scalar control. 


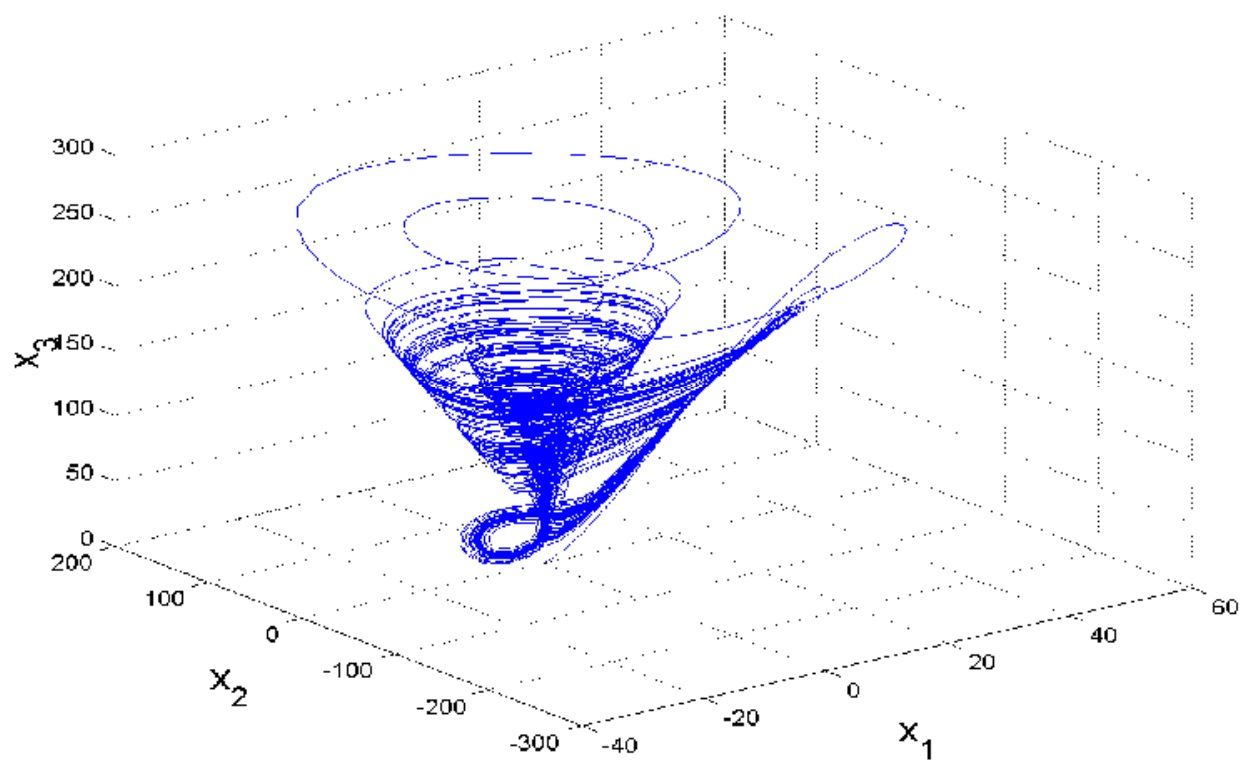

Figure 1. Strange Attractor of the Liu-Liu-Liu-Su Chaotic System

Liu, Liu, Liu and Su (2006) showed that the system (5) has chaotic behaviour when $a=8$, $b=35, c=1.4$ and $u=0$. The strange attractor of the Liu-Liu-Liu-Su chaotic system is illustrated in Figure 1.

In this paper, we consider the output regulation problem for the tracking of constant reference signals (set-point signals).

In this case, the exosystem is given by the scalar dynamics

$$
\dot{\omega}=0
$$

We note that the assumption (H1) of Theorem 1 holds trivially.

Linearizing the dynamics of the Liu-Liu-Liu-Su chaotic system (5) at $x=0$, we obtain

$$
A=\left[\begin{array}{ccc}
-a & a & 0 \\
b & 0 & 0 \\
0 & 0 & -c
\end{array}\right]=\left[\begin{array}{cc}
A_{1} & 0 \\
0 & -c
\end{array}\right] \text { and } B=\left[\begin{array}{l}
0 \\
1 \\
0
\end{array}\right]=\left[\begin{array}{c}
B_{1} \\
0
\end{array}\right]
$$

where

$$
A_{1}=\left[\begin{array}{cc}
-a & a \\
b & 0
\end{array}\right] \text { and } B_{1}=\left[\begin{array}{l}
0 \\
1
\end{array}\right]
$$


International Journal of Computer Science, Engineering and Applications (IJCSEA) Vol.2, No.3, June 2012

From (7) and (8), it follows that the system pair $(A, B)$ is not completely controllable as it has a stable uncontrollable mode at $\lambda=-c$. Since the pair $\left(A_{1}, B_{1}\right)$ is controllable, it follows that the system pair $(A, B)$ is stabilizable. Given any two desired modes $\lambda_{1}, \lambda_{2}<0$, we can use Ackermann's formula to find a matrix $K_{1}=\left[\begin{array}{ll}\alpha & \beta\end{array}\right]$ so that the closed-loop system matrix $A_{1}+B_{1} K_{1}$ will have stable eigenvalues $\lambda_{1}$ and $\lambda_{2}$.

Choosing $K=\left[\begin{array}{ll}K_{1} & 0\end{array}\right]$, it is immediate that the closed-loop system matrix $A+B K$ will have stable eigenvalues $\left\{\lambda_{1}, \lambda_{2},-c\right\}$. This calculation shows that the pair $(A, B)$ is stabilizable.

Hence, the assumption (H2) of Theorem 1 also holds.

Hence, Theorem 1 can be applied to solve the constant regulation problem for the Liu-Liu-Liu-Su chaotic system (5).

\subsection{The Constant Tracking Problem for $x_{1}$}

Here, the tracking problem for the Liu-Liu-Liu-Su chaotic system (5) is given by

$$
\begin{aligned}
& \dot{x}_{1}=a\left(x_{2}-x_{1}\right) \\
& \dot{x}_{2}=b x_{1}-x_{1} x_{3}+x_{1}^{2}+u \\
& \dot{x}_{3}=-c x_{3}+x_{1}^{2} \\
& e=x_{1}-\omega
\end{aligned}
$$

By Theorem 1, the regulator equations of the system (8) are obtained as

$$
\begin{array}{r}
\pi_{2}(\omega)-\pi_{1}(\omega)=0 \\
b \pi_{1}(\omega)-\pi_{1}(\omega) \pi_{3}(\omega)+\pi_{1}^{2}(\omega)+\varphi(\omega)=0 \\
-c \pi_{3}(\omega)+\pi_{1}^{2}(\omega)=0 \\
\pi_{1}(\omega)-\omega=0
\end{array}
$$

Solving the regulator equations (9) for the system (8), we obtain the unique solution as

$$
\begin{aligned}
& \pi_{1}(\omega)=\omega, \quad \pi_{2}(\omega)=\omega, \quad \pi_{3}(\omega)=\frac{1}{c} \omega^{2} \\
& \varphi(\omega)=-b \omega-\omega^{2}+\frac{1}{c} \omega^{3}
\end{aligned}
$$

Using Theorem 1 and the solution (10) of the regulator equations for the system (8), we obtain the following result which provides a solution of the output regulation problem for (8).

Theorem 2. A state feedback control law solving the output regulation problem for the Liu-LiuLiu-Su chaotic system (8) is given by 
International Journal of Computer Science, Engineering and Applications (IJCSEA) Vol.2, No.3, June 2012

$$
u=\varphi(\omega)+K[x-\pi(\omega)]
$$

where $\varphi(\omega), \pi(\omega)$ are defined as in (10) and $K$ is chosen so that $A+B K$ is Hurwitz.

\subsection{The constant Tracking Problem for $x_{2}$}

Here, the tracking problem for the Liu-Liu-Liu-Su chaotic system (5) is given by

$$
\begin{aligned}
& \dot{x}_{1}=a\left(x_{2}-x_{1}\right) \\
& \dot{x}_{2}=b x_{1}-x_{1} x_{3}+x_{1}^{2}+u \\
& \dot{x}_{3}=-c x_{3}+x_{1}^{2} \\
& e=x_{2}-\omega
\end{aligned}
$$

By Theorem 1, the regulator equations of the system (12) are obtained as

$$
\begin{aligned}
\pi_{2}(\omega)-\pi_{1}(\omega) & =0 \\
b \pi_{1}(\omega)-\pi_{1}(\omega) \pi_{3}(\omega)+\pi_{1}^{2}(\omega)+\varphi(\omega) & =0 \\
-c \pi_{3}(\omega)+\pi_{1}^{2}(\omega) & =0 \\
\pi_{2}(\omega)-\omega & =0
\end{aligned}
$$

Solving the regulator equations (13) for the system (12), we obtain the unique solution as

$$
\begin{aligned}
& \pi_{1}(\omega)=\omega, \quad \pi_{2}(\omega)=\omega, \quad \pi_{3}(\omega)=\frac{1}{c} \omega^{2} \\
& \varphi(\omega)=-b \omega-\omega^{2}+\frac{1}{c} \omega^{3}
\end{aligned}
$$

Using Theorem 1 and the solution (14) of the regulator equations for the system (13), we obtain the following result which provides a solution of the output regulation problem for (13).

Theorem 3. A state feedback control law solving the output regulation problem for the Liu-LiuLiu-Su chaotic system (12) is given by

$$
u=\varphi(\omega)+K[x-\pi(\omega)]
$$

where $\varphi(\omega), \pi(\omega)$ are defined as in (14) and $K$ is chosen so that $A+B K$ is Hurwitz.

\subsection{The Constant Tracking Problem for $x_{3}$}

Here, the tracking problem for the Liu-Liu-Liu-Su chaotic system (5) is given by 
International Journal of Computer Science, Engineering and Applications (IJCSEA) Vol.2, No.3, June 2012

$$
\begin{aligned}
& \dot{x}_{1}=a\left(x_{2}-x_{1}\right) \\
& \dot{x}_{2}=b x_{1}-x_{1} x_{3}+x_{1}^{2}+u \\
& \dot{x}_{3}=-c x_{3}+x_{1}^{2} \\
& e=x_{3}-\omega
\end{aligned}
$$

By Theorem 1, the regulator equations of the system (16) are obtained as

$$
\begin{aligned}
\pi_{2}(\omega)-\pi_{1}(\omega) & =0 \\
b \pi_{1}(\omega)-\pi_{1}(\omega) \pi_{3}(\omega)+\pi_{1}^{2}(\omega)+\varphi(\omega) & =0 \\
-c \pi_{3}(\omega)+\pi_{1}^{2}(\omega) & =0 \\
\pi_{3}(\omega)-\omega & =0
\end{aligned}
$$

Solving the regulator equations (17) for the system (16), we obtain the unique solution as

$$
\begin{aligned}
& \pi_{1}(\omega)=\sqrt{c \omega}, \quad \pi_{2}(\omega)=\sqrt{c \omega}, \quad \pi_{3}(\omega)=\omega \\
& \varphi(\omega)=-b \sqrt{c \omega}-c \omega+\omega \sqrt{c \omega}
\end{aligned}
$$

Using Theorem 1 and the solution (18) of the regulator equations for the system (16), we obtain the following result which provides a solution of the output regulation problem for (16).

Theorem 4. A state feedback control law solving the output regulation problem for the Liu-LiuLiu-Su chaotic system (16) is given by

$$
u=\varphi(\omega)+K[x-\pi(\omega)]
$$

where $\varphi(\omega), \pi(\omega)$ are defined as in (18) and $K$ is chosen so that $A+B K$ is Hurwitz.

\section{NUMERical Simulations}

For the numerical simulations, the fourth order Runge-Kutta method with step-size $h=10^{-8}$ is deployed to solve the systems of differential equations using MATLAB.

For simulation, the parameters are chosen as the chaotic case of the Liu-Liu-Liu-Su system, viz. $a=8, \quad b=35$ and $c=1.4$.

For achieving internal stability of the state feedback regulator problem, a feedback gain matrix $K$ must be chosen so that $A+B K$ is Hurwitz.

We note that $\lambda=-c=-1.4$ will always be an uncontrollable mode. We find a feedback gain matrix $K_{1}=\left[\begin{array}{ll}\alpha & \beta\end{array}\right]$ so that the matrix $A_{1}+B_{1} K_{1}$ has stable eigenvalues $\{-5,-5\}$.

Since $\left(A_{1}, B_{1}\right)$ is controllable, we obtain the gain matrix $K_{1}$ by Ackermann's formula (MATLAB) as $K_{1}=\left[\begin{array}{ll}-36.125 & -2\end{array}\right]$. Thus, we have $K=\left[\begin{array}{ll}K_{1} & 0\end{array}\right]=\left[\begin{array}{lll}-36.125 & -2 & 0\end{array}\right]$. 
International Journal of Computer Science, Engineering and Applications (IJCSEA) Vol.2, No.3, June 2012

\subsection{Constant Tracking Problem for $x_{1}$}

Here, the initial conditions are taken as $x_{1}(0)=8, x_{2}(0)=-6, x_{3}(0)=15$ and $\omega=2$.

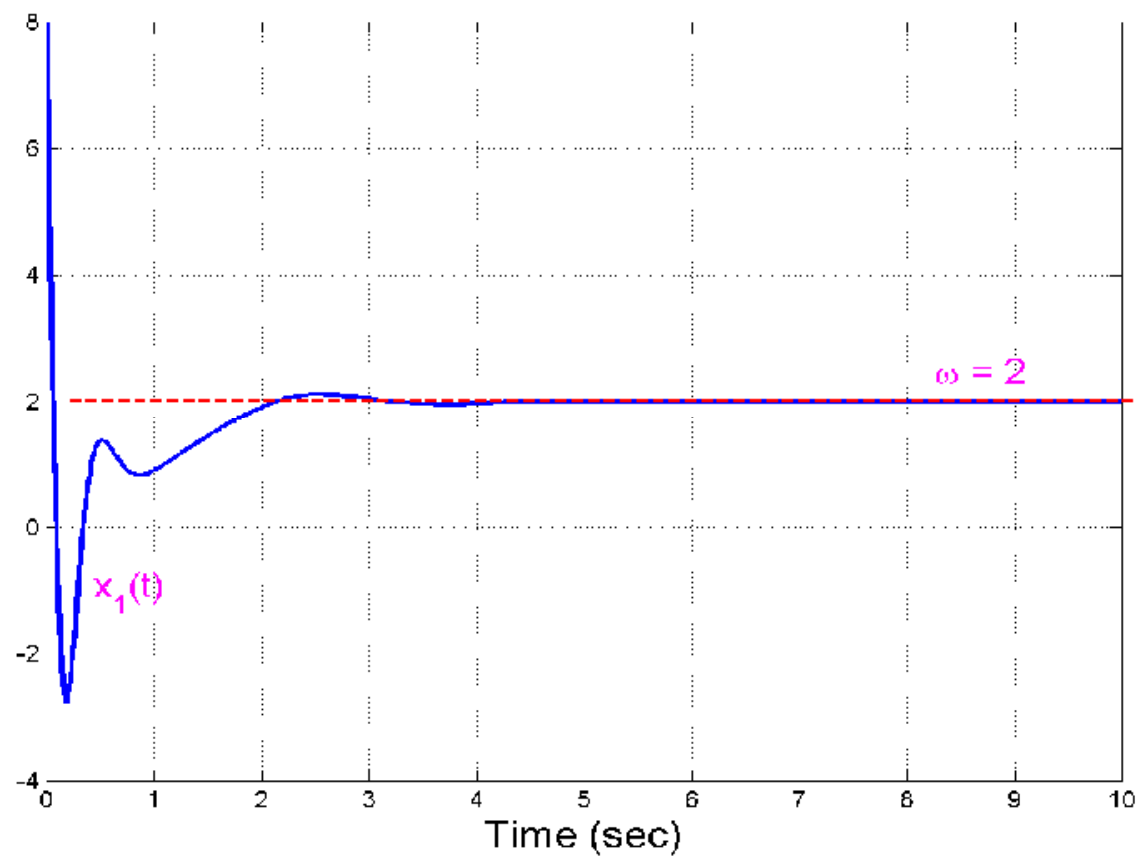

Figure 2. Constant Tracking Problem for $x_{1}$

The simulation graph is depicted in Figure 2 from which it is clear that the state trajectory $x_{1}(t)$ tracks the constant reference signal $\omega=2$ in 4 seconds.

\subsection{Constant Tracking Problem for $x_{2}$}

Here, the initial conditions are taken as $x_{1}(0)=5, x_{2}(0)=9, x_{3}(0)=4$ and $\omega=2$.

The simulation graph is depicted in Figure 3 from which it is clear that the state trajectory $x_{2}(t)$ tracks the constant reference signal $\omega=2$ in 4 seconds. 
International Journal of Computer Science, Engineering and Applications (IJCSEA) Vol.2, No.3, June 2012

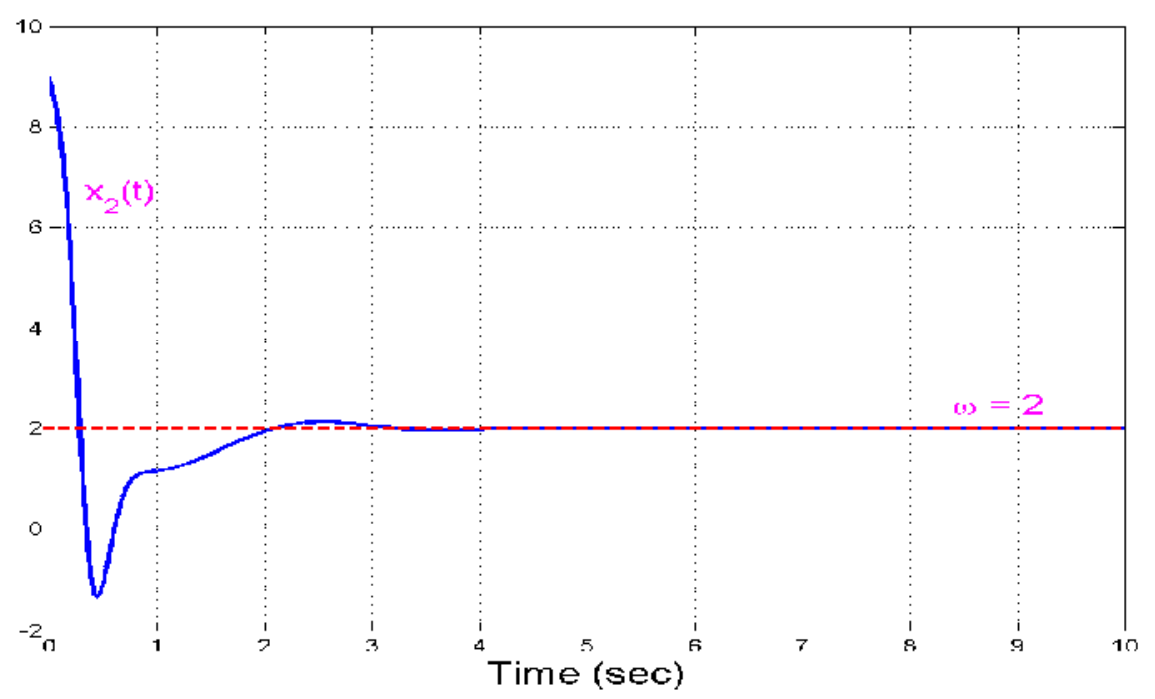

Figure 3. Constant Tracking Problem for $x_{2}$

\subsection{Constant Tracking Problem for $x_{3}$}

Here, the initial conditions are taken as $x_{1}(0)=7, x_{2}(0)=-3, x_{3}(0)=5$ and $\omega=2$.

The simulation graph is depicted in Figure 4 from which it is clear that the state trajectory $x_{3}(t)$ tracks the constant reference signal $\omega=2$ in 4 seconds.

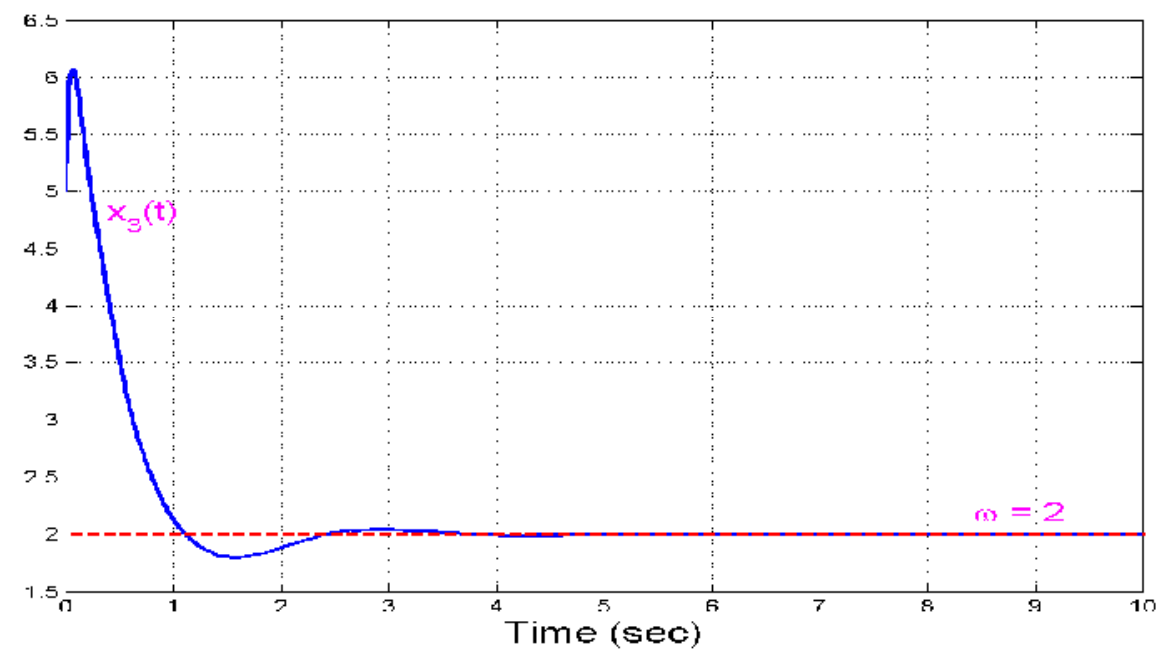

Figure 4. Constant Tracking Problem for $x_{3}$ 


\section{Conclusions}

In this paper, active controller has been designed to solve the output regulation problem for the Liu-Liu-Liu-Su chaotic system (2006) and a complete solution for the tracking of constant reference signals (set-point signals). The state feedback control laws achieving output regulation proposed in this paper were derived using the regulator equations of Byrnes and Isidori (1990). Numerical simulation results were presented in detail to illustrate the effectiveness of the proposed control schemes for the output regulation problem of Liu-Liu-Liu-Su chaotic system to track constant reference signals.

\section{REFERENCES}

[1] Francis, B.A. \& Wonham, W.M. (1975) “The internal model principle for linear multivariable regulators", J. Applied Math. Optimization, Vol. 2, pp 170-194.

[2] Byrnes, C.I. \& Isidori, A. (1990) "Output regulation of nonlinear systems", IEEE Trans. Automatic Control, Vol. 35, pp 131-140.

[3] Carr, J. (1981) Applications of Centre Manifold Theory, Springer Verlag, New York.

[4] Mahmoud, N.A. \& Khalil, H.K. (1996) "Asymptotic regulation of minimum phase nonlinear systems using output feedback", IEEE Trans. Automat. Control, Vol. 41, pp 1402-1412.

[5] Fridman, E. (2003) "Output regulation of nonlinear control systems with delay", Systems \& Control Lett., Vol. 50, pp 81-93.

[6] Chen, Z. \& Huang, J. (2005) "Robust output regulation with nonlinear exosystems", Automatica, Vol. 41, pp 1447-1454.

[7] Chen, Z. \& Huang, J. (2005) “Global robust output regulation for output feedback systems", IEEE Trans. Automat. Control, Vol. 50, pp 117-121.

[8] Liu, L. \& Huang, J. (2008) "Global robust output regulation of lower triangular systems with unknown control direction”, Automatica, Vol. 44, pp. 1278-1284.

[9] Immonen, E. (2007) "Practical output regulation for bounded linear infinite-dimensional state space systems”, Automatica, Vol. 43, pp 786-794.

[10] Pavlov, A., Van de Wouw, N. \& Nijmeijer, H. (2007) "Global nonlinear output regulation: convergence based controller design", Automatica, Vol. 43, pp 456-463.

[11] Xi, Z. \& Ding, Z. (2007) "Global robust output regulation of a class of nonlinear systems with nonlinear exosystems", Automatica, Vol. 43, pp 143-149.

[12] Serrani, A. \& Isidori, A. (2000) "Global robust output regulation for a class of nonlinear systems", Systems \& Control Letters, Vol. 39, pp 133-139.

[13] Serrani, A., Isidori, A. \& Marconi, L. (2000) "Semiglobal output regulation for minimum phase systems", Internat. J. Robust Nonlinear Control, Vol. 10, pp 379-396.

[14] Marconi, L., Isidori, A. \& Serrani, A. (2004) "Non-resonance conditions for uniform observability in the problem of nonlinear output regulation", Systems \& Control Letters, Vol. 53, pp 281-298.

[15] Liu, C., Liu, T., Liu, L. \& Su, Y. (2006) “A new nonlinear chaotic system," International Journal of Nonlinear Sciences and Numerical Simulation, Vol. 7, No. 3, pp. 345-352.

[16] Ogata, K. (1997) Modern Control Engineering, Prentice Hall, New Jersey, U.S.A.

[17] Sundarapandian, V. (2011) "Output regulation of Shimizu-Morioka chaotic system by state feedback control”, International J. Advanced Information Technology, Vol. 1, No. 2, pp. 1-10. 


\section{Author}

Dr. V. Sundarapandian earned his Doctor of Science degree in Electrical and Systems Engineering from Washington University, St. Louis, USA in May 1996. He is a Professor at the R \& D Centre at Vel Tech Dr. RR \& Dr. SR Technical University, Chennai, Tamil Nadu, India. He has published over 260 papers in refereed international journals. He has published over 170 papers in National and International Conferences. He is the Editor-inChief of the AIRCC Journals - International Journal of Instrumentation and Control Systems, International Journal of Control Systems and Computer Modelling, and International Journal of Information Technology, Control and Automation. He is an

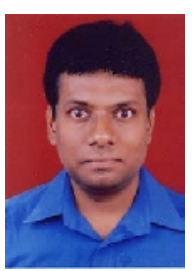
associate editor of many international journals on Computer Science, IT and Control Engineering. His research interests are Linear and Nonlinear Control Systems, Chaos Theory and Control, Soft Computing, Optimal Control, Operations Research, Mathematical Modelling and Scientific Computing. He has delivered several Key Note Lectures on Control Systems, Chaos Theory, Scientific Computing, Mathematical Modelling, MATLAB and SCILAB. 